\title{
Diffusion Thermopower of a Two-Dimensional Hole Gas in SiGe in a Quantum Hall Insulating State
}

\author{
C. Possanzini, ${ }^{1}$ R. Fletcher, ${ }^{2}$ P. T. Coleridge,${ }^{3}$ Y. Feng, ${ }^{3}$ R. L. Williams, ${ }^{3}$ and J.C. Maan ${ }^{1, *}$ \\ ${ }^{1}$ Research Institute for Materials, High Field Magnet Laboratory, University of Nijmegen, Toernooiveld 1, \\ 6525 ED Nijmegen, The Netherlands \\ ${ }^{2}$ Physics Department, Queen's University, Kingston, Ontario, Canada K7L $3 N 6$ \\ ${ }^{3}$ Institute for Microstructural Sciences, National Research Council, Ottawa, Ontario, Canada K1A OR6
}

(Received 1 December 2002; published 28 April 2003)

\begin{abstract}
Both the temperature dependence of resistivity and thermopower of a two-dimensional hole gas in SiGe show a reentrant metal-insulator transition at filling factor $\nu=1.5$, but with strikingly different behavior of the two coefficients. As the temperature is decreased in the insulating state, the resistivity diverges exponentially while the thermopower decreases rapidly, suggesting that the insulating state is due to the presence of a mobility edge rather than a gap at the Fermi energy.
\end{abstract}

PACS numbers: 72.20.Pa, 71.30.+h, 73.23.-b

In a perpendicular magnetic field, two-dimensional systems characterized by short-range carrier scattering frequently exhibit a reentrant metal-insulator transition where an insulating phase appears between two integer quantum Hall states [1-3]. Such insulating states have been observed in $p$-type $\mathrm{Si} / \mathrm{SiGe}$ heterostructures [1] and in $\mathrm{Si}$ metal-oxide-semiconductor field-effect transistors [4], while in GaAs heterostructures, characterized by long-range scattering, an insulating phase appears only between fractional Hall states [5]. In $p$-type $\mathrm{Si} / \mathrm{SiGe}$, this reentrant metal-insulator transition (MIT) at a filling factor $\nu=1.5$ is followed at higher field by a second MIT which occurs in the quantum Hall limit $(\nu<1)$ where the Fermi energy, $E_{\mathrm{F}}$, lies within the lowest Landau level $[1-3,6,7]$. The origin of the MIT at $\nu=$ 1.5 , i.e., an insulating phase in the presence of extended states below $E_{\mathrm{F}}$, is not understood. Recently, a scaling analysis of the magnetoconductivity measurements $[1,2,6]$ demonstrated that the MITs at $\nu=1.5$ and in the quantum limit both scale with the same critical exponent, supporting the idea that both MITs are driven by the same mechanism. However, although scaling behavior is a signature of a phase transition, it does not reveal the origin of the insulating state at $\nu=1.5$.

In this paper, we report thermopower (TEP) measurements on a two-dimensional hole gas in a $\mathrm{Si} / \mathrm{SiGe}$ heterostructure as a function of temperature and magnetic field near the field-induced insulating state at $\nu=1.5$. At low temperatures, TEP is mainly due to diffusion which probes the energy distribution of the carriers. Diffusion TEP is expected to provide more information on the behavior of the density of states around $E_{\mathrm{F}}$. In particular, TEP is expected to answer the question whether the fielddriven insulating phase is the result of an opening of an energy gap at $E_{\mathrm{F}}$ or if it is due to the presence of a disorder-induced mobility gap. In both cases, on decreasing the temperature the resistivity diverges in the insulating state, while the TEP is expected to diverge in the former [8] and to vanish or approach a constant value in the latter [9]. Therefore TEP can distinguish between the two possibilities. In our measurements, TEP does not diverge but tends to a constant at $\nu=1.5$ showing that the insulating phase is due to a mobility gap. This observation may be related to the peculiar many-level structure of a $p$-type $\mathrm{Si} / \mathrm{SiGe}$ heterostructure in a magnetic field, when the Zeeman splitting may exceed the cyclotron energy and the state at $\nu=2$ therefore becomes ferromagnetically polarized. The degeneracy and crossing of Landau levels has been adduced as one of the reasons for the appearance of the insulating state at $\nu=1.5[1,3,10]$.

The present experiments were performed on a strained, asymmetrically doped $\mathrm{Si} / \mathrm{Si}_{0.88} \mathrm{Ge}_{0.12}$ quantum well in which the carriers were confined in a triangular potential well (the sample used in this paper was fabricated from the same wafer as CVD191 in Ref. [11]). By applying a substrate bias, measurements could be made at two different densities: $p=1.9 \times 10^{15} \mathrm{~m}^{-2}$ and $p=2.4 \times$ $10^{15} \mathrm{~m}^{-2}$. The mobilities were strongly temperature dependent [12] and at $1 \mathrm{~K}$ they were 1.3 and $1.5 \mathrm{~m}^{2} / \mathrm{V} \mathrm{s}$, respectively. Conductivity and TEP measurements at zero field show that at the sample densities used here there is no sign of localization (seen for $p<1.0 \times 10^{15} \mathrm{~m}^{-2}$ [11]) and metallic conductivity prevails down to the lowest temperature.

The TEP was measured between two contacts diffused into the ends of the Hall bar which also served as current contacts for resistivity measurements. Measurements of TEP in the insulating state as a function of temperature were made with a Keithley 182 dc voltmeter which provides a good compromise between input impedance, input bias current, and noise. In order to eliminate any spurious voltage caused by the bias current, the signal was measured both with and without temperature gradient, keeping the average temperature of the sample constant [13,14]. Data in swept magnetic fields were obtained with an ac lock-in technique detecting at $4 \mathrm{~Hz}$ 
[15]. The factor used to convert the ac data to absolute thermopower was determined by comparing ac and dc measurements at zero field.

An analysis of both the temperature dependence of TEP at zero field and the temperature and field dependence of the quantum oscillations in the TEP at low fields [12] shows that phonon drag becomes negligible below $0.7 \mathrm{~K}$, leaving diffusion as the dominant driving mechanism at low temperatures. In this Letter, we focus on the behavior at higher fields, especially in the field-induced insulating phase around $\nu=1.5$.

Figure 1 shows the TEP, $S_{x x}$, as a function of magnetic field at various fixed temperatures. In general, previous work on the TEP of many systems in the integer quantum Hall regime leads us to expect that $S_{x x}$ will vanish at integer filling factors and show maxima at half-integer filling factors, analogous to the resistivity [16]. This pattern is followed in the present sample except around $\nu=$ 1.5. Here $S_{x x}$ shows a double peak structure at low temperatures and, at $\nu=1.5$, its magnitude decreases to very small values at the lowest temperatures. For comparison, at the same point the resistivity, $\rho_{x x}$, increases as the temperature decreases indicating an insulating phase.

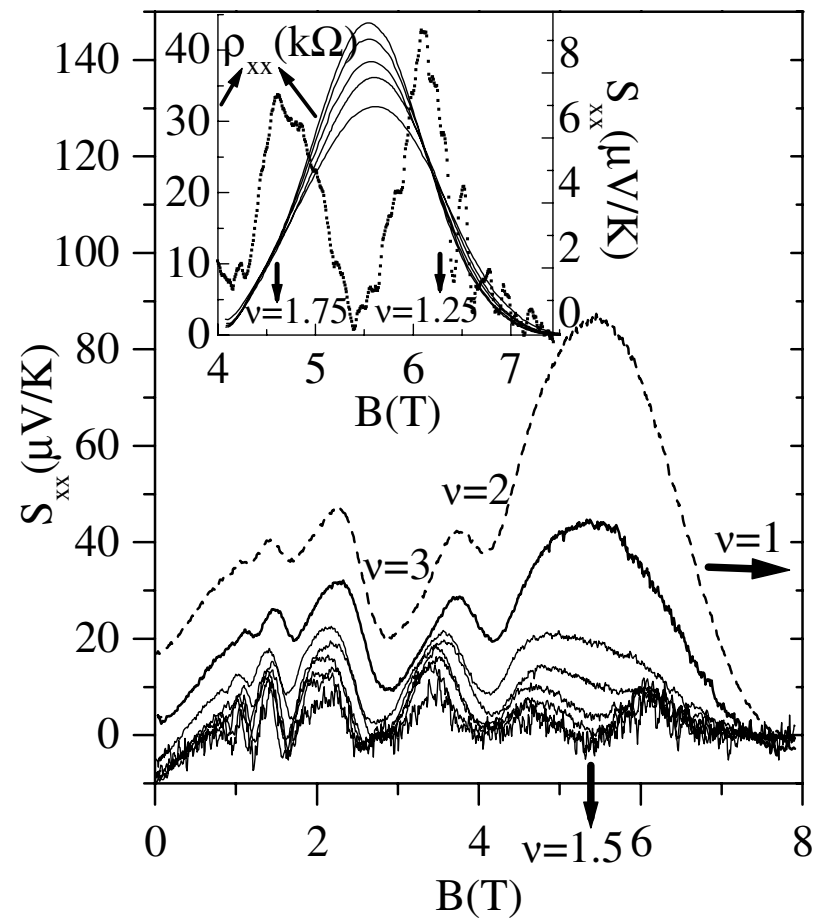

FIG. 1. The thermopower, $S_{x x}$, as a function of magnetic field at temperatures of $0.32,0.43,0.50,0.63,0.76,0.89,1.07$, and $1.23 \mathrm{~K}$. The dashed line is $S_{x x}$ at the highest temperature $(1.23 \mathrm{~K})$. The inset shows resistivity measurements (solid line) at $0.3,0.4,0.5,0.6$, and $0.8 \mathrm{~K}$, and thermopower (dotted line which is a smoothed version of the curve of $S_{x x}$ at $0.3 \mathrm{~K}$ shown in the main panel) as a function of magnetic field around $\nu=1.5$. The sample density is $p=1.9 \times 10^{15} \mathrm{~m}^{-2}$.
Figure 2 shows the detailed temperature dependences of $S_{x x}$ and $\rho_{x x}$ at $\nu=1.5$ for two different densities: $p=$ $1.9 \times 10^{15} \mathrm{~m}^{-2}$ and $p=2.4 \times 10^{15} \mathrm{~m}^{-2}$. The graph clearly shows that in the insulating state the resistivity diverges at low temperatures, while the TEP appears to vanish. The low temperature peaks in $S_{x x}$ around $\nu=1.5$ shown in Fig. 1 at approximately $4.7 T$ and $6.2 T$ occur at $\nu=1.25$ and 1.75 and correspond to the "critical magnetic fields," where $\rho_{x x}$ is temperature independent. They separate the metallic phases $(\nu<1.25$ and $\nu>1.75)$ from the insulating phase $(1.25<\nu<1.75)$ [6]. In the resistivity shown in the inset of Fig. 1, this is particularly clear at $\nu=1.25$, but at $\nu=1.75$ it is blurred by the proximity of a separate integer quantum Hall effect transition $[1,6]$, and a sharp crossing point can be resolved only at lower temperatures. Thus, the transition from the metal to the insulating state is directly seen in $S_{x x}$, but in a completely different manner from $\rho_{x x}$.

To illustrate the difference in behavior between TEP in the various states, in Fig. 3 we plot $S_{x x}$ as a function of temperature for four different filling factors: $\nu=2.5$ (metallic), $\nu=1.25$ and 1.75 (critical), and $\nu=1.5$ (insulating). At low temperatures $S_{x x}$ clearly decreases more rapidly in the insulating state than in the other states. At higher temperatures, the rise of $S_{x x}$ is undoubtedly due to phonon drag, $S_{x x}^{g}$. However, here we concentrate on the behavior of $S_{x x}$ at low temperatures where diffusion dominates.

For metallic samples, when the cyclotron energy $\hbar \omega_{c}$ exceeds the broadening of the Landau level $\Gamma$ (low disorder), $S_{x x}^{d}$ is directly related to the entropy of the partially filled Landau levels. When the spin splitting is resolved, $S_{x x}^{d}$ is determined by the entropy $S=k_{\mathrm{B}} \ln W$, where $W=N ! /(f N) !(N-f N)$ !, with $N$ the number of states in a spin-resolved level and $f$ the fractional filling

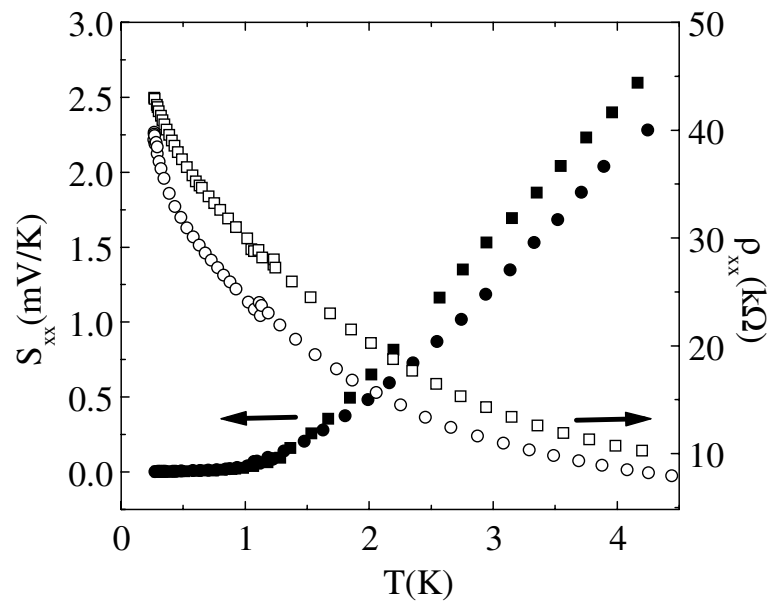

FIG. 2. The temperature dependence of the resistivity (open symbols) and thermopower (solid symbols) at $\nu=1.5$ for two different densities: $p=1.9 \times 10^{15} \mathrm{~m}^{-2}(\square)$ and $p=$ $2.4 \times 10^{15} \mathrm{~m}^{-2}(\bigcirc)$. 


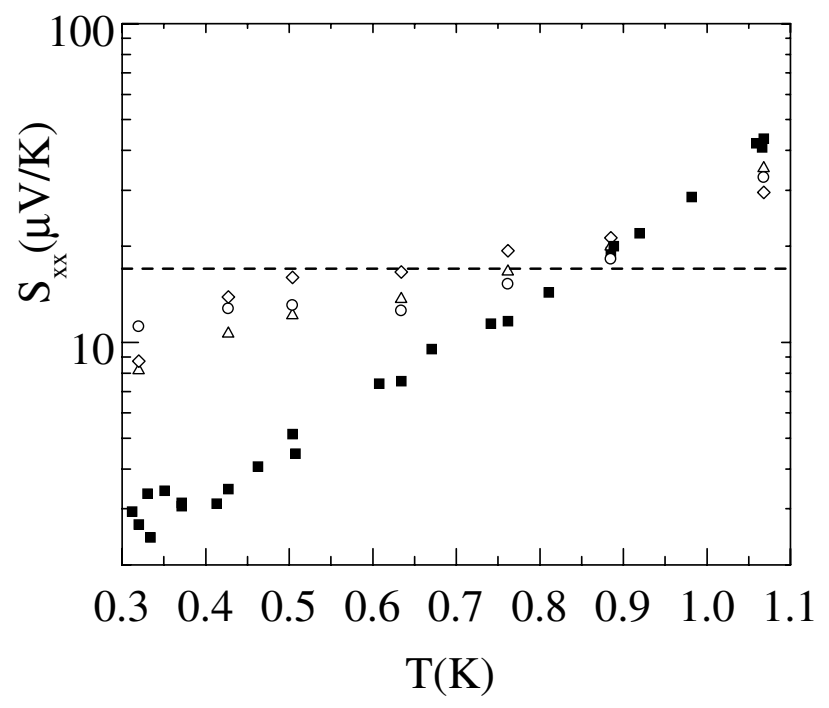

FIG. 3. Temperature dependence of $S_{x x}$ in the insulating phase at $\nu=1.5(\square)$, in the critical states at $\nu=1.75(\triangle)$ and $\nu=1.25(\bigcirc)$, and in the metal state at $\nu=2.5(\diamond)$. The dashed line is the theoretical entropy limit at $\nu=2.5$.

of the last level. The total charge is $N \nu e$ and, e.g., at half filling where $k_{\mathrm{B}} \ln W=k_{\mathrm{B}} N \ln 2$, the TEP is maximum and is given by the entropy per unit of charge, i.e., $S_{x x}^{d}=$ $k_{\mathrm{B}} \ln 2 / \nu e$ [17].

When $k_{\mathrm{B}} T<\Gamma$, the expected values become a function of $k_{\mathrm{B}} T / \Gamma$ and decrease with decreasing temperature [18]. The measured quantum mobility of this sample at low magnetic fields is found to be $1.4 \mathrm{~m}^{2} / \mathrm{V} \mathrm{s}$ at $2.4 \times$ $10^{15} \mathrm{~m}^{-2}$ [11], giving $k_{\mathrm{B}} T=\Gamma$ at $T \approx 1.65 \mathrm{~K}$, and reducing the calculated values of the maxima of $S_{x x}$ by a factor of about 0.7 at $0.3 \mathrm{~K}$. However, $\Gamma$ is known to increase as $B^{1 / 2}$ at high fields so that this reduction factor will be an underestimate. On the basis of these arguments, we estimate $S_{x x}$ at $\nu=1.25,1.75$, and 2.5 to be 27,19 , and $17 \mu \mathrm{V} / \mathrm{K}$, respectively, which are consistent with the observed values in Fig. 3 of about $10 \mu \mathrm{V} / \mathrm{K}$ at $0.3 \mathrm{~K}$. The previous argument is not appropriate for the insulating phase at $\nu=1.5$, and indeed the experimental value of $S_{x x}$ at low temperature is $2-3 \mu \mathrm{V} / \mathrm{K}$, an order of magnitude smaller than the estimated value of $S_{x x}=$ $28 \mu \mathrm{V} / \mathrm{K}$ based on the entropy result.

Previous work has been inconclusive about whether the MIT at $\nu=1.5$ should be attributed to an opening of a many body gap at $E_{\mathrm{F}}$, or to $E_{\mathrm{F}}$ being located in localized states below a mobility edge. At zero magnetic field it is known that $S^{d}$ can distinguish these two cases, and we believe that the results remain valid in arbitrary fields as long as $\rho_{x x} \gg \rho_{y x}$ as here [1]. When $E_{\mathrm{F}}$ is located in a gap, $S^{d} \sim T^{-1}$ and $S^{d}$ should therefore diverge at low temperatures. Such behavior has previously been seen in $p$-type GaAs heterostructures $[19,20]$ and in Pd and Pd-Au films [9]. However, our experimental results clearly show that $S_{x x}$ becomes very small as $T \rightarrow 0$, contrary to the expected behavior due to the opening of a gap.
On the other hand, a finite $S^{d}$ at low temperatures is expected when carriers conduct by hopping between localized states below a mobility edge. For variable range hopping (VRH), theory predicts an activated behavior of the resistivity $\rho_{x x}(T)=\rho_{c} \exp \left(T_{\circ} / T\right)^{n}$, where $n=$ $1 / 3$ if the density of states is finite at $E_{\mathrm{F}}$ (Mott VRH) and $n=1 / 2$ if a soft Coulomb gap opens up at $E_{\mathrm{F}}$ (EfrosShklovskii VRH) [21]. The corresponding results for TEP are $S^{d} \sim T^{1 / 3}$ for the Mott model and $S^{d}$ becomes constant with a Coulomb gap.

We now focus on a more detailed analysis of our data in the insulating state. In Fig. 4, we show $S_{x x}$ as a function of temperature at $\nu=1.5$ for the same two densities as in Fig. 3. The temperature dependence can be described by either $S_{x x}=\alpha T^{1 / 3}+\eta T^{4}$ (Mott VRH shown as the dotted line in Fig. 4) or $S_{x x}=\gamma+\eta T^{4}$ (Efros-Shklovskii VRH, solid line in Fig. 4), with $\alpha=18 \mu \mathrm{V} / \mathrm{K}^{4 / 3}$ and $\gamma=2.5 \mu \mathrm{V} / \mathrm{K}$.

The term $T^{4}$, with $\eta=35 \mu \mathrm{V} / \mathrm{K}^{5}$, describes the residual contribution of the phonon drag, dominant at higher temperatures $(T>0.6 \mathrm{~K})$. At low temperatures when carriers are localized, theory predicts $S^{g}$ to be zero because drag requires the conservation of crystal momentum which cannot hold for electron scattering by phonons between localized states [19,22]. Andreev et al. [23] recently reported that $S^{g}$ vanished in a bulk doped Ge sample at zero field in the hopping regime. Therefore, regardless of the particular model for the MIT in the present case, we might have anticipated a rapid drop in $S_{x x}^{g}$ in the insulating phase as the temperature decreases.

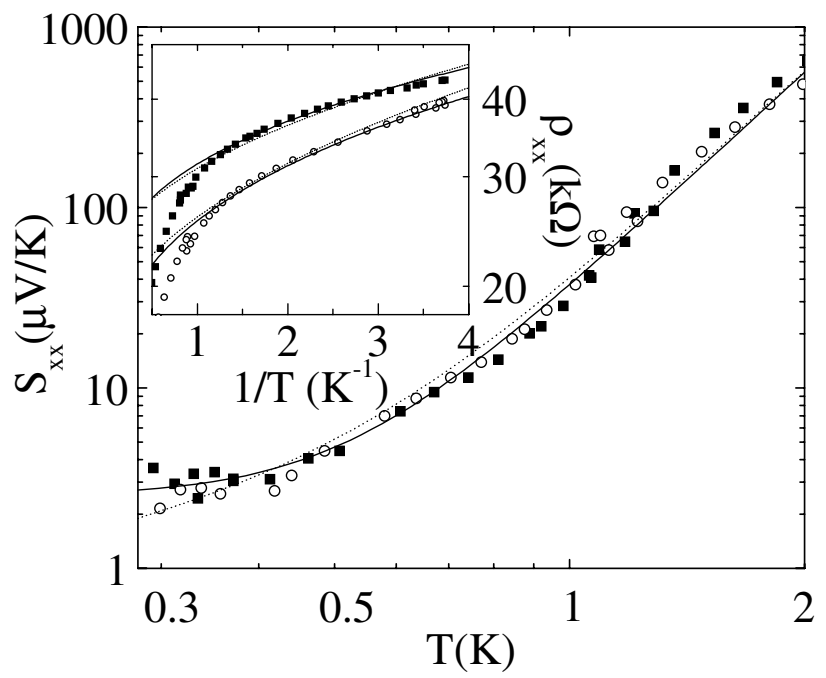

FIG. 4. The thermopower measured at $\nu=1.5$ as a function of temperature for two different densities: $p=1.9 \times 10^{15} \mathrm{~m}^{-2}$ (a) and $p=2.4 \times 10^{15} \mathrm{~m}^{-2}(\bigcirc)$. The dotted line is the fit $S_{x x}=\alpha T^{1 / 3}+\eta T^{4}$ (Mott VRH), and the solid line is the fit $S_{x x}=\gamma+\eta T^{4}$ (Efros-Shklovskii VRH). Resistivity measurements are shown for the same two densities (same symbols as for TEP) in the inset. The solid line is the fit to the EfrosShklovskii model and the dotted line the fit to the Mott model. 
Drag would still be possible via carriers excited above the mobility gap but this would presumably show activated behavior. We see no strong evidence for this, the weaker $T^{4}$ dependence apparently being followed to the lowest temperatures.

In the localization regime, the calculated magnitudes of $S^{d}$ [9] for both the Efros-Shklovskii $(\gamma)$ and Mott $(\alpha)$ VRH models depend linearly on the asymmetry of the density of states, $D(E)$ at $E_{\mathrm{F}}$, i.e., on the factor $[\partial \ln D(E) / \partial E]_{E_{\mathrm{F}}}$. Using $\Gamma$ as the energy scale, we have $[\partial \ln D(E) / \partial E]_{E_{\mathrm{F}}} \sim 1 / \Gamma$ which leads to the estimates $\alpha=32 \mu \mathrm{V} / \mathrm{K} 4 / 3$ and $\gamma=2.21 ; \mu \mathrm{V} / \mathrm{K}$ in keeping with the experimental values of $18 \mu \mathrm{V} / \mathrm{K}^{4 / 3}$ and $2.5 \mu \mathrm{V} / \mathrm{K}$, respectively.

In conclusion, we have measured the thermopower of a $p$-type $\mathrm{Si} / \mathrm{SiGe}$ heterostructure as a function of magnetic field and temperature. The thermopower reflects the metal-insulator transition previously probed by magnetoresistance measurements [1-3,5-7], but in a totally different manner. The combined data on the resistivity and thermopower at $\nu=1.5$ suggest that the insulating state is not due to an opening of an energy gap at the Fermi energy, but it is caused by a mobility gap.

This work is part of a research program of the Stichting voor Fundamenteel Onderzoek der Materie (FOM), financially supported by N.W.O. (The Netherlands). The work was also supported by the Natural Sciences and Engineering Research Council of Canada.

*Electronic address: maan@sci.kun.nl

[1] P.T. Coleridge, A.S. Sachrajda, P. Zawadski, R. L. Williams, and H. Lafointaine, Solid State Commun. 102, 755 (1997); P.T. Coleridge, P. Zawadski, A. S. Sachrajda, R. L. Williams, and Y. Feng, Physica (Amsterdam) 6E, 268 (2000).

[2] R. B. Dunford et al., J. Phys. Condens. Matter 9, 1565 (1997); R. B. Dunford, N. Griffin, M. Pepper, C. J. Emeleus, P. J. Phillips, and T. E. Whall, Physica (Amsterdam) 6E, 297 (2000).

[3] M. R. Sakr, M. Rahimi, S. V. Kravchenko, P. T. Coleridge, R. L. Williams, and J. Lapointe, Phys. Rev. B 64, 161308(R) (2001).
[4] S.V. Kravchenko, J. A. A. J. Perenboom, and V. M. Pudalov, Phys. Rev. B 44, 13513 (1985).

[5] T. Sajoto, Y. P. Li, L.W. Engel, D.C. Tsui, and M. Shayegan, Phys. Rev. Lett. 70, 2321 (1993).

[6] C. Possanzini, L. Ponomarenko, D. de Lang, A. de Visser, S. M. Olsthoorn, R. Fletcher, Y. Feng, P. T. Coleridge, R L. Williams, and J.C. Maan, Physica (Amsterdam) 12E, 600 (2002).

[7] M. Hilke, D. Shahar, S. H. Song, D. C. Tsui, Y. H. Xie, and D. Monroe, Nature (London) 395, 675 (1998).

[8] B. L. Gallagher and P. N. Butcher, in Handbook on Semiconductors, edited by P.T. Landsberg (Elsevier, Amsterdam, 1992), Vol. 1, p. 817.

[9] M. J. Burns and P. M. Chaikin, Phys. Rev. B 27, 5924 (1983); M. J. Burns, Phys. Rev. B 40, 5473 (1989).

[10] F. F. Fang, P. J. Wang, B. S. Meyerson, J. J. Nocera, and K. E. Ismail, Surf. Sci. 263, 175 (1992).

[11] P. T. Coleridge, R. L. Williams, Y. Feng, and P. Zawadzki, Phys. Rev. B 56, R12 764 (1997).

[12] C. Possanzini, R. Fletcher, Y. Feng, P. T. Coleridge, R. L. Williams, and J. C. Maan (to be published).

[13] R. Fletcher, V. M. Pudalov, A. D. B. Radcliffe, and C. Possanzini, Semicond. Sci. Technol. 16, 386 (2001).

[14] R. Fletcher, P. T. Coleridge, and Y. Feng, Phys. Rev. B 52, 2823 (1995).

[15] B. Tieke, R. Fletcher, U. Zeitler, M. Henini, and J.C. Maan, Phys. Rev. B 58, 2017 (1998).

[16] In general $E_{\mathrm{F}}$ is always located in localized states at integer filling factors. However, although $S^{d}$ and $S^{g}$ do appear to go to zero at these points, because $\rho_{x x} \gg \rho_{y x}$ the situation is not the same as in the usual case at zero magnetic field or in the present case.

[17] H. Oji, Phys. Rev. B 29, 3148 (1984).

[18] W. Zawadski and R. Lassnig, Surf. Sci. 142, 225 (1984).

[19] V. Bayot, X. Ying, M. B. Santos, and M. Shayegan, Europhys. Lett. 25, 613 (1994).

[20] V. Bayot, E. Grivei, H.C. Manoharan, X. Ying, and M. Shayegan, Phys. Rev. B 52, R8621 (1995).

[21] D. G. Polyakov and B. I. Shklovskii, Phys. Rev. B 48, 11167 (1993); Phys. Rev. Lett. 70, 3796 (1993).

[22] I. P. Zvyagin, in Hopping Transport in Solids, edited by M. Pollak and B. Shklovskii (North-Holland, Amsterdam, 1991).

[23] A. G. Andreev, A. G. Zabrodskii, S. V. Egorov, and I. P. Zvyagin, Sov. Phys. Semicond. 31, 1008 (1997). 